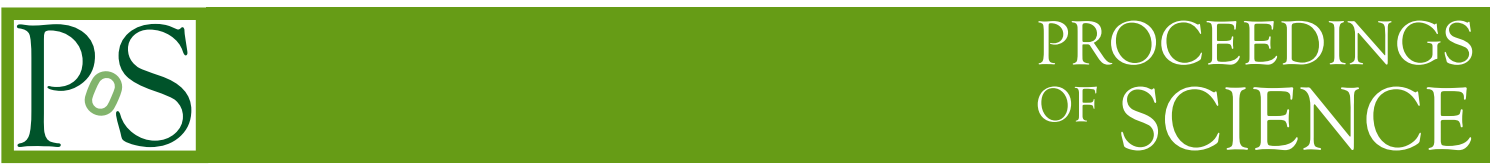

\title{
$Z_{c}(3900)$ from coupled-channel HAL QCD approach on the lattice
}

\section{Yoichi lkeda*}

Theoretical Research Division,Nishina Center, RIKEN, Saitama 351-0198, Japan

E-mail: yikeda@riken.jp

for HAL QCD Collaboration

Hadrons to Atomic nuclei

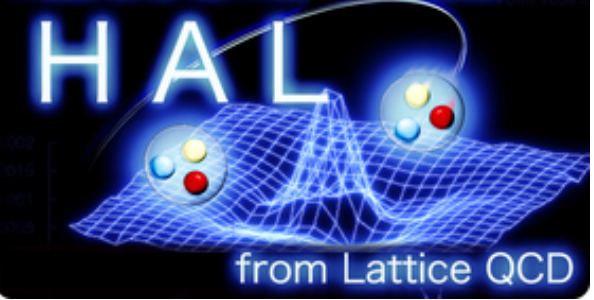

The structure of the $Z_{c}(3900)$ is studied from dynamical lattice simulations of QCD at $m_{\pi} \simeq$ $410 \mathrm{MeV}$. The s-wave two-body coupled-channel scatterings in $I^{G}\left(J^{P C}\right)=1^{+}\left(1^{+-}\right)$are carried out on the lattice using the $\pi J / \psi-\rho \eta_{c}-\bar{D} D^{*}$ coupled-channel potentials derived from the HAL QCD approach. The calculated invariant mass spectra of the $\pi J / \psi$ and $\bar{D} D^{*}$ show enhancements just above the $\bar{D} D^{*}$ threshold energy due to the strong transition potentials between the $\pi J / \psi$ and $\bar{D} D^{*}$. The poles of the two-body amplitudes are also examined and are found far below the $\bar{D} D^{*}$ threshold energy on the second Riemann sheets, so that the peak corresponding to the $Z_{c}(3900)$ in the invariant mass spectra is naturally explained by not a conventional resonance but a coupled-channel kinematical effects.

The 33rd International Symposium on Lattice Field Theory

14 - 18 July 2015

Kobe International Conference Center, Kobe, Japan

\footnotetext{
* Speaker.
} 


\section{Introduction}

One of long standing challenges in hadron physics is to look for the exotic nature of hadrons other than ordinary mesons(quark-antiquark states) and baryons(three-quark states) on the basis of the quantum chromodynamics (QCD). One of good targets is the $Z_{c}(3900)$ in $I^{G}\left(J^{P C}\right)=1^{+}\left(1^{+-}\right)$, which has been recently reported by BESIII [1] and Belle [2] Collaborations and confirmed by CLEO-c Collaboration [3]. The $Z_{c}(3900)$ is found as a peak in $\pi^{ \pm} J / \psi$ invariant mass spectra of the final states produced by the $e^{+} e^{-}$collisions. Since the $Z_{c}(3900)$ has a electric charge, at least four quarks $(c \bar{c} u \bar{d})$ are dynamically involved.

The resonance structure of the $Z_{c}(3900)$ is theoretically studied using phenomenological tetraquark, $\pi J / \psi$ - and $\bar{D} D^{*}$-molecule models (see, e.g., Refs. 44 5]). While the threshold kinematical effects for the peak corresponding to the $Z_{c}(3900)$ observed in the experiments are discussed by the initial-state-pion-exchange mechanism [6] and the $\bar{D} D^{*}$ threshold cusp [7] in the $\pi J / \psi$ $\bar{D} D^{*}$ coupled-channel scattering. However, due to lack of information of the $\pi J / \psi-\bar{D} D^{*}$ coupledchannel interactions, predictions for the structure of the $Z_{c}(3900)$ has not been settled yet, so that the lattice QCD (LQCD) studies are highly awaited to conclude the $Z_{c}(3900)$ structure. LQCD studies to search for the $Z_{c}(3900)$ in $I^{G}\left(J^{P C}\right)=1^{+}\left(1^{+-}\right)$have been performed using the conventional approach [8 9 10], in which the spectrum on the lattice is constructed from the temporal correlations of four-quark and two-meson correlation functions, and no isolated eigenstate from meson-meson scattering states is found. These results could indicate that the $Z_{c}(3900)$ may not be an ordinary resonance state. However, in order to answer why the peak in the $\pi J / \psi$ invariant mass spectrum is observed in the experiments, LQCD simulations with manifest coupled-channel scatterings are necessary.

In this study, we investigate the structure of the $Z_{c}(3900)$ in $I^{G}\left(J^{P C}\right)=1^{+}\left(1^{+-}\right)$using the HAL QCD approach [11, 12, 13, 14, 15, 16]. In the HAL QCD approach, asymptotic scattering wave functions are defined from the spatial correlations of hadron four point correlation functions. Then potentials which are faithful to QCD S-matrix are derived from LQCD simulations 17, 18, 19, 20, 21, 22, 23, 24]. The HAL QCD potentials are used for various applications such as the properties of nuclei and exotic hadrons [25, 26, 27, 28, 29]. Since the extension to coupled-channel scatterings is straightforward [15, 16, 30, 31], we apply the coupled-channel HAL QCD approach to derive the swave $\pi J / \psi-\rho \eta_{c}-\bar{D} D^{*}$ coupled-channel potentials. Equipped with the coupled-channel potentials, we calculate the invariant mass spectra of the $\pi J / \psi$ and $\bar{D} D^{*}$, and examine the pole positions of the $Z_{c}(3900)$ on the complex energy plane.

This paper is organized as follows. In Sec. 2, we present the HAL QCD approach to extract coupled-channel potentials between two mesons and then, in Sec. 3, show the numerical setup of our lattice QCD simulations. In Sec. 4 , we show our numerical results for the s-wave $\pi J / \psi-\rho \eta_{c^{-}}$ $\bar{D} D^{*}$ coupled-channel potentials, the invariant mass spectra of the $\pi J / \psi$ and $\bar{D} D^{*}$ and the pole positions. Sec. 5 is devoted to discussions and a summary.

\section{HAL QCD approach for coupled-channel scatterings}

In this section, we show the basic equations to derive coupled-channel potentials in the HAL 
QCD approach [15, 16, 30, 31]. We start with the two-meson correlation functions defined as

$$
\begin{aligned}
C^{a b}(\vec{r}, t) & \equiv \sum_{\vec{x}}\left\langle 0\left|\phi_{1}^{a}(\vec{x}+\vec{r}, t) \phi_{2}^{a}(\vec{x}, t) \overline{\mathscr{J}}^{b}\left(t_{\text {src. }}=0\right)\right| 0\right\rangle \\
& =\sum_{n} A_{n}^{b} \sqrt{Z_{1}^{a} Z_{2}^{a}} \psi_{n}^{a}(\vec{r}) e^{-W_{n} t}+\ldots
\end{aligned}
$$

with $A_{n}^{b}=\left\langle n\left|\overline{\mathscr{J}}^{b}\left(t_{\text {src. }}=0\right)\right| 0\right\rangle$ and $\sqrt{Z_{1}^{a} Z_{2}^{a}} \psi_{n}^{a}(\vec{r})=\sum_{\vec{x}}\left\langle 0\left|\phi_{1}^{a}(\vec{x}+\vec{r}, t) \phi_{2}^{a}(\vec{x}, t)\right| n\right\rangle$ with $Z_{i}^{a}(i=1$, 2 ) being the wave function renormalization factors for mesons $i$. The superscripts $a$ and $b$ denote the flavor channel $\left(a, b=\pi J / \psi, \rho \eta_{c}, \bar{D} D^{*}\right)$. $\overline{\mathscr{J}}^{a}\left(t_{\text {src. }}=0\right)$ stands for a zero momentum wall source operator at $t_{\text {src. }}=0$ which creates two-meson states in channel $a$, and $\phi_{1,2}^{a}$ is a point-like interpolating sink operator for mesons in channel $a . \psi_{n}^{a}(\vec{r})$ is the equal-time NambuBethe-Salpeter (NBS) wave function in channel $a$ for $n$-th eigenstate $|n\rangle$ with the relativistic energy $W_{n}=\sqrt{\left(m_{1}^{a}\right)^{2}+\left(\vec{k}_{n}^{a}\right)^{2}}+\sqrt{\left(m_{2}^{a}\right)^{2}+\left(\vec{k}_{n}^{a}\right)^{2}}$, and ellipses represent inelastic contributions that we do not explicitly consider.

Let us consider $t$ sufficiently large that the contributions from the $\pi J / \psi, \rho \eta_{c}$ and $\bar{D} D^{*}$ scattering states and possible bound states remain while those from inelastic states such as the $\bar{D}^{*} D^{*}$ become negligible. Then, from the NBS wave function, we define coupled-channel energy-independent potentials [12,14,16] as

$$
\begin{array}{r}
H_{0}^{a} \psi_{n}^{a}(\vec{r})+\sum_{b} \int d \overrightarrow{r^{\prime}} U^{a b}\left(\vec{r}, \vec{r}^{\prime}\right) \psi_{n}^{b}\left(\overrightarrow{r^{\prime}}\right)=E_{n}^{a} \psi_{n}^{a}(\vec{r}), \\
U^{a b}\left(\vec{r}, \overrightarrow{r^{\prime}}\right)=\sum_{n, n^{\prime}}\left(E_{n}^{a}-H_{0}^{a}\right) \psi_{n}^{a}(\vec{r}) \cdot \tilde{\psi}_{n^{\prime}}^{b *}\left(\overrightarrow{r^{\prime}}\right),
\end{array}
$$

for all eigenstates $n$, where $H_{0}^{a}=-\nabla^{2} / 2 \mu^{a}$ with $\mu^{a}=m_{1}^{a} m_{2}^{a} /\left(m_{1}^{a}+m_{2}^{a}\right)$ and $E_{n}^{a}=\left(\vec{k}_{n}^{a}\right)^{2} / 2 \mu^{a}$ is a non-relativistic energy. $\tilde{\psi}_{n}^{a *}(\vec{r})$ is the dual basis of the NBS wave function $\psi_{n}^{a}(\vec{r})$ and satisfies $\int d \vec{r} \tilde{\psi}_{n}^{a *}(\vec{r}) \psi_{n^{\prime}}^{b}(\vec{r})=\delta_{n n^{\prime}} \delta_{a b}$

Since the coupled-channel HAL QCD potentials $U^{a b}\left(\vec{r}, \overrightarrow{r^{\prime}}\right)$ are energy-independent, normalized correlation functions $R^{a b}(\vec{r}, t) \equiv C^{a b}(\vec{r}, t) e^{\left(m_{1}^{a}+m_{2}^{a}\right) t} / \sqrt{Z_{1}^{a} Z_{2}^{a}}$ satisfy [13, 16

$$
\left(-\frac{\partial}{\partial t}-H_{0}^{a}\right) R^{a b}(\vec{r}, t) \simeq \sum_{n} A_{n}^{b}\left(E_{n}^{a}-H_{0}^{a}\right) \psi_{n}^{a}(\vec{r}) e^{-\Delta W_{n}^{a} t}=\sum_{c} \int d \overrightarrow{r^{\prime}} \Delta^{a c} U^{a c}\left(\vec{r}, \overrightarrow{r^{\prime}}\right) R^{c b}\left(\overrightarrow{r^{\prime}}, t\right),
$$

where the non-relativistic approximation that $\Delta W_{n}^{a} \equiv W_{n}-m_{1}^{a}-m_{2}^{a}=E_{n}^{a}+O\left(\left(k_{n}^{a}\right)^{4} /\left(m_{1}^{a}\right)^{3},\left(k_{n}^{a}\right)^{4} /\left(m_{2}^{a}\right)^{3}\right)$ is used, and $\Delta^{a c}$ denote $\Delta^{a c}=e^{\left(m_{1}^{a}+m_{2}^{a}\right) t} / e^{\left(m_{1}^{c}+m_{2}^{c}\right) t}$ to subtract the threshold energy. This approximation is not necessary if we allow time derivatives of higher order in Eq. (2.4), which, however, are found negligible for the systems investigated in this paper. By projecting $R^{a b}(\vec{r}, t)$ onto s-wave states using $A_{1}^{+}$representation of the cubic group, we finally obtain the leading order s-wave potentials of the velocity expansion as

$$
\left(-\frac{\partial}{\partial t}-H_{0}^{a}\right) R^{a b}(\vec{r}, t)=\sum_{c} \Delta^{a c} V_{\mathrm{LO}}^{a c}(\vec{r}) R^{c b}(\vec{r}, t) .
$$

\section{Numerical setup of LQCD simulations}

In order to extract the s-wave meson-meson potentials in Eq. (2.5) from LQCD simulation, we employ (2+1)-flavor full QCD gauge configurations generated by the PACS-CS collaboration [32, 33] on a $32^{3} \times 64$ lattice with the renormalization group improved gauge action at $\beta=$ 
1.90 and the non-perturbative $O(a)$-improved Wilson quark action $\left(C_{\mathrm{SW}}=1.715\right)$ at $\left(\kappa_{u d}, \kappa_{s}\right)=$ $(0.13754,0.13640)$. The parameters correspond to the lattice cutoff $a^{-1}=2176 \mathrm{MeV}$ (lattice spacing $a=0.0907$ (13) fm leading to the spatial lattice volume $\left.L^{3} \simeq(2.9 \mathrm{fm})^{3}\right)$. For the charm quark, we employ a relativistic heavy quark (RHQ) action [34], which is designed to remove the leading and next-to-leading order cutoff errors associated with heavy charm quark mass, $\mathscr{O}\left(\left(m_{c} a\right)^{n}\right)$ and $\mathscr{O}\left(\left(m_{c} a\right)^{n}\left(a \Lambda_{Q C D}\right)\right)$, respectively. In our simulations, we take the same parameters as in Ref. [35]. The meson masses obtained from the simulation are listed in Table 1 .

\begin{tabular}{cccccc}
\hline \hline$m_{\pi}$ & $m_{\rho}$ & $m_{\eta_{c}}$ & $m_{J / \psi}$ & $m_{\bar{D}}$ & $m_{D^{*}}$ \\
\hline $411(1)$ & $896(8)$ & $2988(1)$ & $3097(1)$ & $1903(1)$ & $2056(3)$ \\
\hline \hline
\end{tabular}

Table 1: Meson masses obtained in this study in $\mathrm{MeV}$ unit.

\section{Coupled-channel potentials and structure of the $Z_{c}(3900)$ in $I^{G}\left(J^{P C}\right)=1^{+}\left(1^{+-}\right)$}

In Fig. 11 we show the results of the s-wave $\pi J / \psi-\rho \eta_{c}-\bar{D} D^{*}$ coupled-channel potentials. We find that all diagonal potentials $\left(V_{\pi J / \psi-\pi J / \psi}, V_{\rho \eta_{c}-\rho \eta_{c}}\right.$ and $\left.V_{\bar{D} D^{*}-\bar{D} D^{*}}\right)$ are weak (Fig. 1 (a)). This observation indicates that the $Z_{c}(3900)$ is neither simple $\pi J / \psi$ nor $\bar{D} D^{*}$ molecule. For the offdiagonal potentials (Fig. 1 (b)), we find the weak transition potential between the $\pi J / \psi$ and the $\rho \eta_{c}$ channels $\left(V_{\pi J / \psi-\rho \eta_{c}}\right)$. This observation is consistent with the heavy quark spin symmetry, in which the charm quark spin flip amplitude is suppressed by $\mathscr{O}\left(1 / m_{c}\right)$. While in the $\bar{D} D^{*}-\pi J / \psi$ and $\bar{D} D^{*}-\rho \eta_{c}$ channels, we find the strong transition potentials $\left(V_{\bar{D} D^{*}-\pi J / \psi}\right.$ and $\left.V_{\bar{D} D^{*}-\rho \eta_{c}}\right)$.

(a)

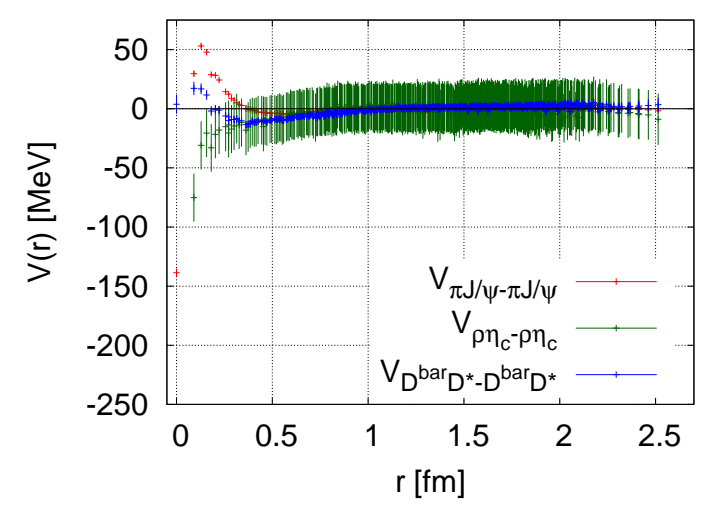

(b)

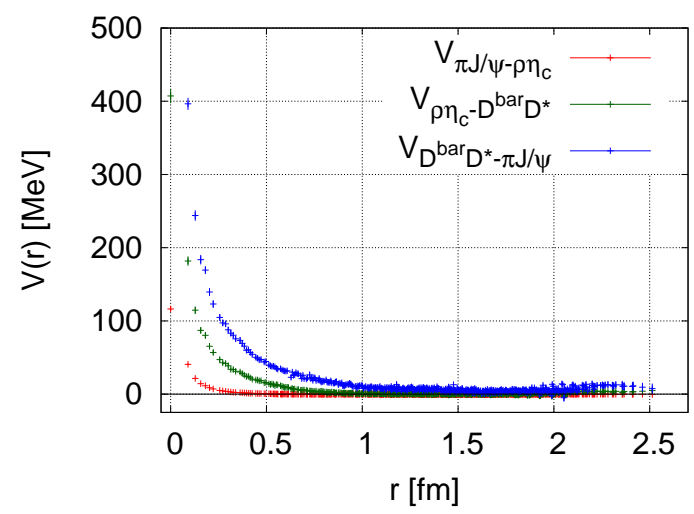

Figure 1: (Color online). Left panel (a) for the s-wave diagonal potentials in the $\pi J / \psi-\pi J / \psi$ (red), $\rho \eta_{c^{-}}$ $\rho \eta_{c}$ (green) and $\bar{D} D^{*}-\bar{D} D^{*}$ (blue) channels. Right panel (b) for the s-wave off-diagonal potentials in the $\pi J / \psi-\rho \eta_{c}$ (red), $\rho \eta_{c}-\bar{D} D^{*}$ (green) and $\bar{D} D^{*}-\pi J / \psi$ (blue) channels. The coupled-channel potentials are obtained at $m_{\pi} \simeq 410 \mathrm{MeV}$.

To investigate whether derived coupled-channel potentials can produce the peak structure in the $\pi J / \psi$ mass spectrum, we study the s-wave $\pi J / \psi-\rho \eta_{c}-\bar{D} D^{*}$ coupled-channel two-body scattering by solving the Schödinger equation in the infinite volume and calculate the $\pi J / \psi$ and $\bar{D} D^{*}$

\footnotetext{
${ }^{1}$ It is noticed that the physical thresholds below the $\bar{D} D^{*}$ are the $\pi J / \psi, \pi \pi \eta_{c}$ and $\pi \psi^{\prime}$. However, due to the heavy $m_{\pi}$ in our simulation, the $\pi \psi^{\prime}$ goes above the $\bar{D} D^{*}$ threshold, and the $\rho \rightarrow \pi \pi$ decay is not allowed with $L \simeq 3 \mathrm{fm}$.
} 
invariant mass spectrum from the two-body amplitudes. Fig. 2 (a) and (b) show the resultant invariant mass spectra in the $\pi J / \psi$ and $\bar{D} D^{*}$ channels, respectively. In the $\pi J / \psi$ invariant mass spectrum, we find that the peak structure just above the $\bar{D} D^{*}$ threshold energy, which corresponds to $3959 \mathrm{MeV}$ for $m_{\pi} \simeq 410 \mathrm{MeV}$. This peak is produced by the large coupling between the $\pi J / \psi$ and the $\bar{D} D^{*}$ channels. When we turn off the off-diagonal potentials, no peak structure is observed as shown in Fig. 2 (a).

(a)

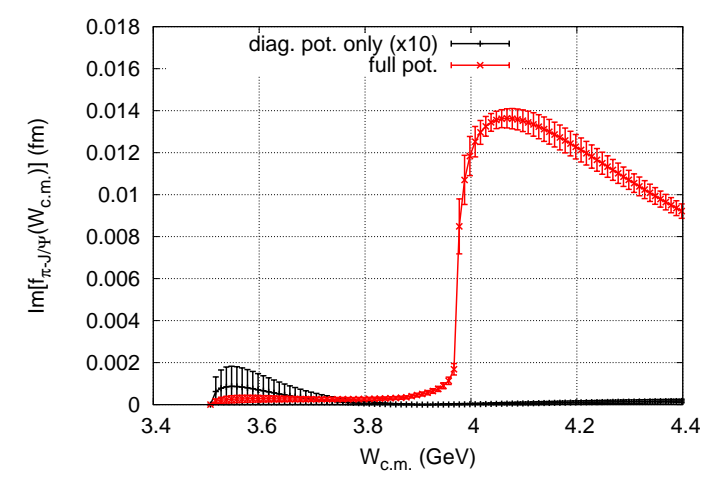

(b)

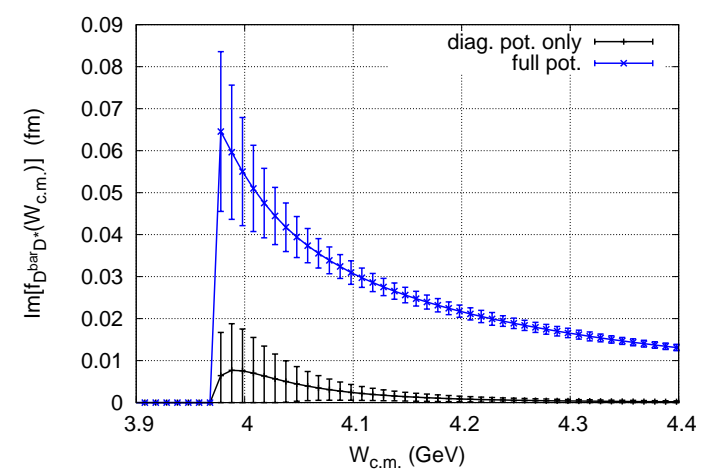

Figure 2: (Color online). The two-body invariant mass spectra in the $\pi J / \psi$ (a) and $\bar{D} D^{*}$ (b) channels. In both panels (a) and (b), the black curves show the invariant mass spectra calculated without the off-diagonal potentials. In the $\pi J / \psi$ channel (a), the factor of ten is multiplied for the result without channel couplings.

The line shape of the $\bar{D} D^{*}$ invariant mass spectrum in Fig. 2 (b) indicates the threshold cusp due to the strong $\pi J / \psi-\bar{D} D^{*}$ channel coupling. To check whether the cusp corresponds to the nature of the $Z_{c}(3900)$, we examine the pole positions on the complex energy plane. Fig. 3] shows the two-body $\pi J / \psi-\pi J / \psi$ amplitude on the second Riemann sheets for all the $\pi J / \psi, \rho \eta_{c}$ and $\bar{D} D^{*}$ channels. Although we find the poles of the amplitude, the poles are located about $150 \mathrm{MeV}$ below the $\bar{D} D^{*}$ threshold energy. These poles are not conventional resonance poles but virtual poles, and the contributions from the poles to the two-body scattering are suppressed. Therefore, we conclude that the experimentally observed $Z_{c}(3900)$ state is not a conventional resonance state but a coupled-channel cusp.

\section{Summary}

We have studied the s-wave $\pi J / \psi-\rho \eta_{c}-\bar{D} D^{*}$ coupled-channel interactions in $I^{G}\left(J^{P C}\right)=1^{+}\left(1^{+-}\right)$, using (2+1)-flavor full QCD gauge configurations generated at $m_{\pi} \simeq 410 \mathrm{MeV}$, in order to investigate the structure of the $Z_{c}(3900)$. For the charm quark, we have employed the relativistic heavyquark action to account for its proper dynamics on the lattice.

The s-wave coupled-channel energy-independent potentials are extracted from Nambu-BetheSalpeter wave functions using the coupled-channel HAL QCD approach. The coupled-channel HAL QCD potentials are then used to calculate scattering observables such as invariant mass spectra and poles on the complex energy plane. All the diagonal potentials in $\pi J / \psi, \rho \eta_{c}$ and $\bar{D} D^{*}$ channels are found to be weak. Therefore, the structure of the $Z_{c}(3900)$ is not a simple $\pi J / \psi$ or 


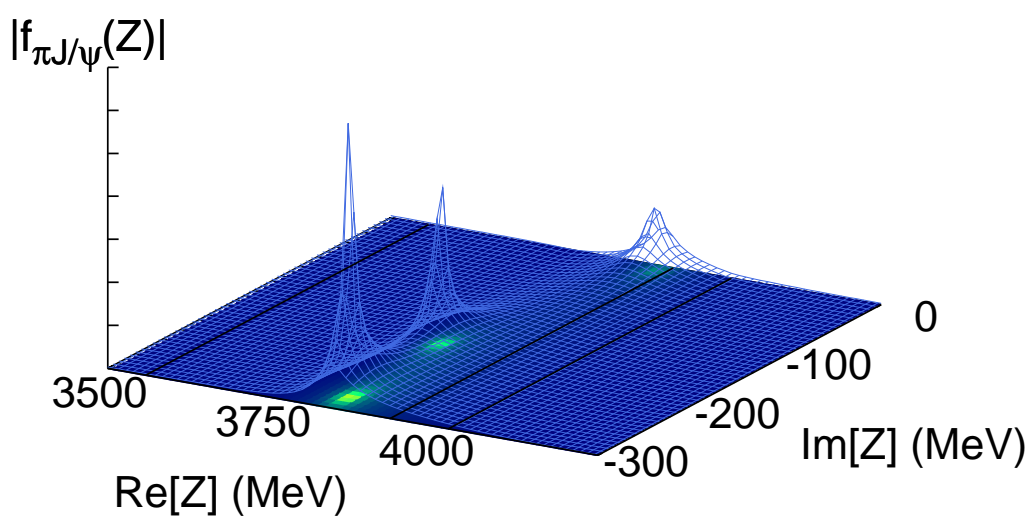

Figure 3: (Color online). The two-body $\pi J / \psi-\pi J / \psi$ amplitude on the complex energy plane. The result on the second sheets for all the $\pi J / \psi, \rho \eta_{c}$ and $\bar{D} D^{*}$ channels is shown.

$\bar{D} D^{*}$ molecule. The $\pi J / \psi-\rho \eta_{c}$ transition potential is found to be weak, which is consistent with the heavy quark spin symmetry. On the other hand, the strong transition potentials between the $\pi J / \psi$ and $\bar{D} D^{*}$ channels are found. Due to this strong $\pi J / \psi-\bar{D} D^{*}$ coupling, the peak just above the $\bar{D} D^{*}$ threshold is observed in the two-body $\pi J / \psi$ invariant mass spectrum. Meanwhile, the line shape of the $\bar{D} D^{*}$ invariant mass spectrum indicate that the peak in the $\pi J / \psi$ invariant mass spectrum could be produced by the $\bar{D} D^{*}$ threshold cusp. In order to check whether the peak corresponds to the threshold cusp, pole positions are calculated, and the virtual poles are found. Since the poles are located far below the $\bar{D} D^{*}$ threshold, our LQCD results indicate that the $Z_{c}(3900)$ is not a conventional resonance but a coupled-channel threshold cusp.

\section{Acknowledgment}

The authors thank Drs. T. Matsuki, M. Oka, S. Takeuchi, M. Takizawa and S. Yasui for fruitful discussions. We also thank the PACS-CS Collaboration and ILDG/JLDG [36] for providing us with full QCD gauge configurations used in this study. Numerical calculations were carried out on NECSX9 and SX8R at Osaka University and SR16000 at YITP in Kyoto University. This project is supported in part by by Grant-in-Aid for Scientific Research on Innovative Areas(No.2004:20105001, 20105003) and for Scientific Research(No. 25800170), and SPIRE (Strategic Program for Innovative REsearch).

\section{References}

[1] M. Ablikim et al. [BESIII Collaboration], Phys. Rev. Lett. 110 (2013) 252001; Phys. Rev. Lett. 112 (2014) 022001. 
[2] Z.Q. Liu et al. [Belle Collaboration], Phys. Rev. Lett. 110 (2013) 252002.

[3] T. Xiao et al. [CLEO-c Collaboration], Phys. Lett. B 727 (2013) 366.

[4] M. B. Voloshin, Phys. Rev. D 87 (2013) 9, 091501.

[5] M. Cleven et al., Phys. Rev. D 92 (2015) 1, 014005.

[6] D. Y. Chen, X. Liu and T. Matsuki, Phys. Rev. D 88 (2013) 3, 036008.

[7] E. S. Swanson, Phys. Rev. D 91 (2015) 3, 034009.

[8] S. Prelovsek and L. Leskovec, Phys. Lett. B 727 (2013) 172.

[9] S. H. Lee et al. [Fermilab Lattice and MILC Collaborations], PoS LATTICE 2014 (2014) 125.

[10] S. Prelovsek, C. B. Lang, L. Leskovec and D. Mohler, Phys. Rev. D 91 (2015) 1, 014504.

[11] N. Ishii, S. Aoki and T. Hatsuda, Phys. Rev. Lett. 99 (2007) 022001.

[12] S. Aoki, T. Hatsuda and N. Ishii, Prog. Theor. Phys. 123 (2010) 89.

[13] N. Ishii et al. [HAL QCD Collaboration], Phys. Lett. B 712 (2012) 437.

[14] S. Aoki et al. [HAL QCD Collaboration], PTEP 2012 (2012) 01A105.

[15] S. Aoki et al. [HAL QCD Collaboration], Proc. Japan Acad. B 87 (2011) 509.

[16] S. Aoki et al., Phys. Rev. D 87 (2013) 3, 034512.

[17] H. Nemura, N. Ishii, S. Aoki and T. Hatsuda, Phys. Lett. B673 (2009) 136.

[18] T. Inoue et al. [HAL QCD Collaboration], Prog. Theor. Phys. 124 (2010) 591.

[19] T. Inoue et al. [HAL QCD Collaboration], Phys. Rev. Lett. 106 (2011) 162002.

[20] K. Murano, N. Ishii, S. Aoki and T. Hatsuda, Prog. Theor. Phys. 125 (2011) 1225.

[21] T. Inoue et al. [HAL QCD Collaboration], Nucl. Phys. A 881 (2012) 28.

[22] T. Doi et al. [HAL QCD Collaboration], Prog. Theor. Phys. 127 (2012) 723.

[23] K. Murano et al. [HAL QCD Collaboration], Phys. Lett. B 735 (2014) 19.

[24] T. Kurth, N. Ishii, T. Doi, S. Aoki and T. Hatsuda, JHEP 1312 (2013) 015.

[25] T. Inoue et al. [HAL QCD Collaboration], Phys. Rev. Lett. 111 (2013) 11, 112503.

[26] Y. Ikeda et al. [HAL QCD Collaboration], Phys. Lett. B 729 (2014) 85.

[27] F. Etminan et al. [HAL QCD Collaboration], Nucl. Phys. A 928 (2014) 89.

[28] T. Inoue et al. [HAL QCD Collaboration], Phys. Rev. C 91 (2015) 1, 011001.

[29] M. Yamada et al. [HAL QCD Collaboration], PTEP 2015 (2015) 7, 071B01.

[30] K. Sasaki et al. [HAL QCD Collaboration], PTEP 2015 113B01, arXiv:1504.01717 [hep-lat].

[31] Y. Ikeda et al., arXiv:1602.03465 [hep-lat].

[32] S. Aoki et al. [PACS-CS Collaboration], Phys. Rev. D 79 (2009) 034503.

[33] S. Aoki et al. [PACS-CS Collaboration], Phys. Rev. D 81 (2010) 074503.

[34] S. Aoki, Y. Kuramashi and S. -i. Tominaga, Prog. Theor. Phys. 109 (2003) 383.

[35] Y. Namekawa et al. [PACS-CS Collaboration], Phys. Rev. D 84 (2011) 074505.

[36] International Lattice Data Grid, http://www.lqcd.org/ildg; Japan Lattice Data Grid, http://.jldg.org 